\title{
Diagnostic accuracy of nucleic acid amplification tests (NAATs) in urine for genitourinary tuberculosis: a systematic review and meta-analysis
}

\author{
Carlos Altez-Fernandez ${ }^{1 *}$, Victor Ortiz ${ }^{1}$, Majid Mirzazadeh², Luis Zegarra ${ }^{3}$, Carlos Seas ${ }^{4,5}$ and Cesar Ugarte-Gil ${ }^{1,4,6,7}$
}

\begin{abstract}
Background: Genitourinary tuberculosis is the third most common form of extrapulmonary tuberculosis. Diagnosis is difficult because of unspecific clinical manifestations and low accuracy of conventional tests. Unfortunately, the delayed diagnosis impacts the urinary tract severely. Nucleic acid amplification tests yield fast results, and among these, new technologies can also detect drug resistance. There is lack of consensus regarding the use of these tests in genitourinary tuberculosis; we therefore aimed to assess the accuracy of nucleic acid amplification tests in the diagnosis of genitourinary tuberculosis and to evaluate the heterogeneity between studies.

Methods: We did a systematic review and meta-analysis of research articles comparing the accuracy of a reference standard and a nucleic acid amplification test for diagnosis of urinary tract tuberculosis. We searched Medline, EMBASE, Web of Science, LILACS, Cochrane Library, and Scopus for articles published between Jan 1, 1990, and Apr 14, 2016. Two investigators identified eligible articles and extracted data for individual study sites. We analyzed data in groups with the same index test. Then, we generated pooled summary estimates ( $95 \% \mathrm{Cls})$ for sensitivity and specificity by use of random-effects meta-analysis when studies were not heterogeneous.
\end{abstract}

Results: We identified eleven relevant studies from ten articles, giving information on PCR, LCR and Xpert MTB/RIF tests. All PCR studies were "in-house" tests, with different gene targets and had several quality concerns therefore we did not proceed with a pooled analysis. Only one study used LCR. Xpert studies were of good quality and not heterogeneous, pooled sensitivity was 0.87 (0.66-0.96) and specificity was 0.91 (0.84-0.95).

Conclusion: PCR studies were highly heterogeneous. Among Xpert MTB/RIF studies, specificity was favorable with an acceptable confidence interval, however new studies can update meta-analysis and get more precise estimates. Further high-quality studies are urgently needed to improve diagnosis of genitourinary tuberculosis.

Protocol registration: PROSPERO CRD42016039020.

Keywords: Genitourinary tuberculosis, Nucleic acid amplification test, Systematic review

\section{Background}

Tuberculosis (TB) is still one of the world's biggest threats to public health. In 2014, TB killed 1.5 million people and was the single infectious disease leading cause of death worldwide [1]. The World Health Organization (WHO) has estimated that only in 2014

\footnotetext{
* Correspondence: carlos.altez.f@upch.pe

${ }^{1}$ Facultad de Medicina Alberto Hurtado, Universidad Peruana Cayetano Heredia, Av. Honorio Delgado 430, Urb. Ingeniería, S.M, P Lima, Perú

Full list of author information is available at the end of the article
}

there were 9.6 million new TB cases, $12 \%$ of which were HIV-positive [1].

Extrapulmonary TB (EPTB) accounts for approximately 10 to $20 \%$ of all the $\mathrm{TB}$ affected population. Genitourinary TB (GUTB) is the third most common location of EPTB, after pleural and lymph node involvement [2]. In addition, concomitant GUTB and pulmonary TB is found in $2-10 \%$ and in $15-20 \%$ of patients in developed and developing countries, respectively [2]. 
GUTB is defined as the infection by Mycobacterium tuberculosis of the urinary tract, the male genitalia or the female genitalia, however, most authors refer to GUTB for reporting only the first [3-5]. The infection occurs in most cases after long periods of latent infection followed by hematogenous spread to the kidneys, epididymis or fallopian tubes; prostate seeding has also been reported but is extremely rare. Other genitourinary organs are affected by local spread [5-7].

Clinical manifestations of GUTB are non-specific, depending on the organs affected and the severity of their involvement, and can mimic several urologic and gynecologic diseases [8]. Diagnosing GUTB is difficult and often overlooked. Conventional diagnostic tests include urine microscopy and culture. Since GUTB is usually paucibacillary [9], most samples evaluated by microscopy are negative. Although culture is the gold standard for diagnosing GUTB, currently available culture methods, including both solid and liquid platforms are insensitive for diagnosing GUTB, and show a number of disadvantages including long-turn around times, high contamination rates and cost [10].

Furthermore, delayed diagnosis may result in worse outcomes, such as infertility, non-functioning unilateral kidney, contracted bladder and even renal failure [8]. Case series reported that $26.9 \%$ of patients on average had a non-functioning unilateral kidney, $7.4 \%$, renal failure and $10 \%$ had contracted bladder at the time of diagnosis of genitourinary tuberculosis $[2,4]$.

Nucleic acid amplification tests (NAATs), have been developed to overcome the limitations of conventional tests in TB [11-15]. These can yield results in between 2 to $48 \mathrm{~h}$ [16] NAATs can be classified as commercial or in-house ("home-brew") tests [16] They can be classified, as well, by their mechanism: polymerase chain reaction (PCR) tests, ligase chain reaction(LCR) tests and more recent technologies (variants of PCR) such as Xpert MTB/RIF and Genotype ${ }^{\circ}$ MTBDRplus [17, 18].

While the use of NAAT has been well studied in pulmonary TB, their use in GUTB lacks enough highquality evidence $[11,19,20]$. Therefore, we conducted this systematic review to determine the accuracy of NAAT performed in urine for diagnosing GUTB and to identify the factors related to heterogeneity between studies of the result.

\section{Methods}

We developed our study in accordance with the Cochrane's group guidelines and methods for systematic reviews and meta-analyses of diagnostic tests [21]. The protocol was registered at PROSPERO database with the number CRD42016039020.

\section{Search strategy and selection criteria}

We searched Medline, EMBASE, Scopus, Lilacs, Cochrane Library and Web of Science for studies in all languages published from January 1, 1990, to April 14, 2016 (see Additional file 1: Appendix S1 for search terms).

We included all the studies that compared a NAAT in urine for diagnosis of GUTB with the gold standard. These studies should have presented data in the form of true positives (TP), true negatives (TN), false positives (FP) and false negatives (FN) In case insufficient data were reported, authors were contacted via email to provide additional information regarding their published reports.

We included studies that used a microbiological or a broad standard reference as gold standard. We define the microbiological standard reference as a positive mycobacterial culture on solid or liquid media for one or more specimens from each patient. We define a broad standard reference as either a microbiological standard reference or clinical manifestations of the disease with clinical response after appropriate treatment. We excluded 3 studies that showed results fewer than ten patients, as such results are at high risk of bias. A total of 13 patients were excluded using this criterion. We also excluded studies that had a case-control design, as these may lead to overestimation of accuracy in diagnostic studies.

Two reviewers (CA and VO) independently screened the collected citations for relevance and revised full-text articles with pre-specified eligibility criteria; disagreements about study selection were resolved by consultation with a third reviewer (CU-G). The results of the literature search are presented in a flowchart following the PRISMA guidelines (Fig. 1) [22].

\section{Data extraction and quality appraisal}

$\mathrm{CA}$ and $\mathrm{VO}$ extracted data and resolved disagreements by consensus (Additional file 1). We assessed study quality with the Quality Assessment Tool for Diagnostic Accuracy Studies (QUADAS-2) [23]. QUADAS-2 consists of four domains: patient selection, index test, reference standard, and flow and timing. We assessed all domains for the potential for risk of bias and the first three domains for concerns regarding applicability. We used questions, called signaling questions, for each domain to form judgments about the risk of bias. As recommended, we first developed guidance on how to evaluate each signaling question and interpret this information tailored to this review. Then, one review author (CA) piloted the tool with four of the included studies. Based on experience gained from the pilot study, we finalized the tool (see Additional file 1: Appendix S2 for quality appraisal). Two authors (CA and $\mathrm{VO}$ ) independently assessed the methodological quality of the included 


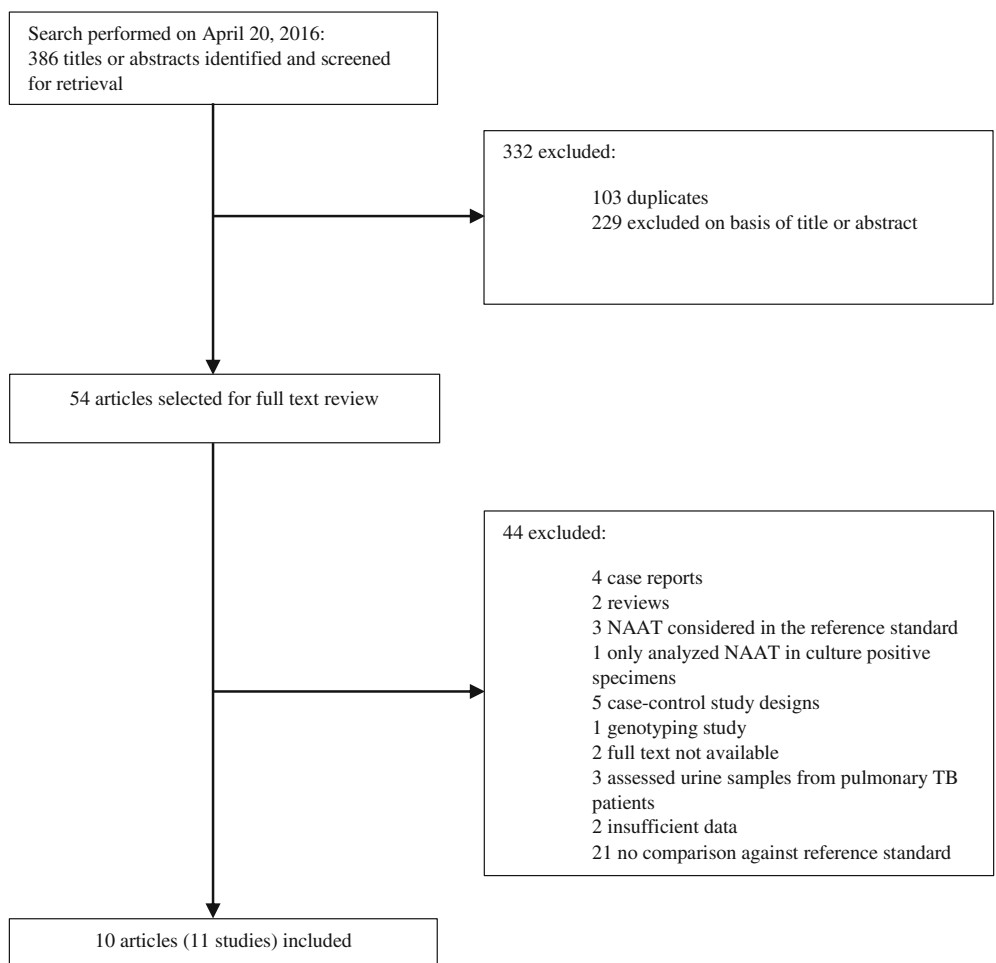

Fig. 1 Selection of studies reporting on the use of a NAAT for GUTB diagnosis in urine

studies with the finalized tool; disagreements about methodological quality were resolved by consultation with a third reviewer (CU-G).

\section{Statistical analysis}

We used standard methods recommended for metaanalyses of diagnostic studies [21] Analyses were done using the $\mathrm{R}$ statistical programming language version 3 $[24,25]$.

To minimize projected heterogeneity, we decided a priori to analyze data in certain subgroups: studies using PCR, studies using LCR and studies using Xpert MTB/ RIF. For each subgroup, we assessed heterogeneity visually with forest plots and summary receiver-operating characteristic (SROC) curves with 95\% prediction regions, and statistically with $\chi^{2}$ statistics. Regarding the forest plots, we used a statistic continuity correction of $5 \%$ applied to the reported sensitivity and specificity. This correction affected studies with large confidence intervals and extreme values, we then used these results for further analysis as recommended for meta-analyses of diagnostic studies [21]. We generated pooled summary estimates and differences for sensitivity and specificity with $95 \%$ confidence intervals by using a bivariate summary ROC curve [26]. It is assumed that sensitivity and specificity vary across studies because of differences in study populations, sampling errors, and differences in implicit thresholds applied to the data to separate patients. Thus, a random-effect model was applied to account for between-study heterogeneity.

\section{Results}

We identified 386 citations, of which 54 were potentially relevant based on the title and abstract (Fig. 1). After full-text review, ten articles were included in our analysis, providing data of eleven studies addressing different NAATs.

\section{Study characteristics}

Of the eleven studies selected, eight studies used PCR, one study used LCR and, two studies used Xpert MTB/ RIF. All of the PCR studies were "in-house" tests. The average size of each study was 248 (specimens or subjects), with a range of 20 to 1000 . Table 1 outlines the main characteristics of these studies.

Overall, nine studies were done in middle-income countries were TB burden is high. Only one study collected data retrospectively. Furthermore, a microbiological reference standard was used in eight studies, one used a broad reference standard and the other two used different combinations of clinical manifestations and culture.

Each of the studies that used PCR had a unique protocol for running the test. Six studies used a single PCR 


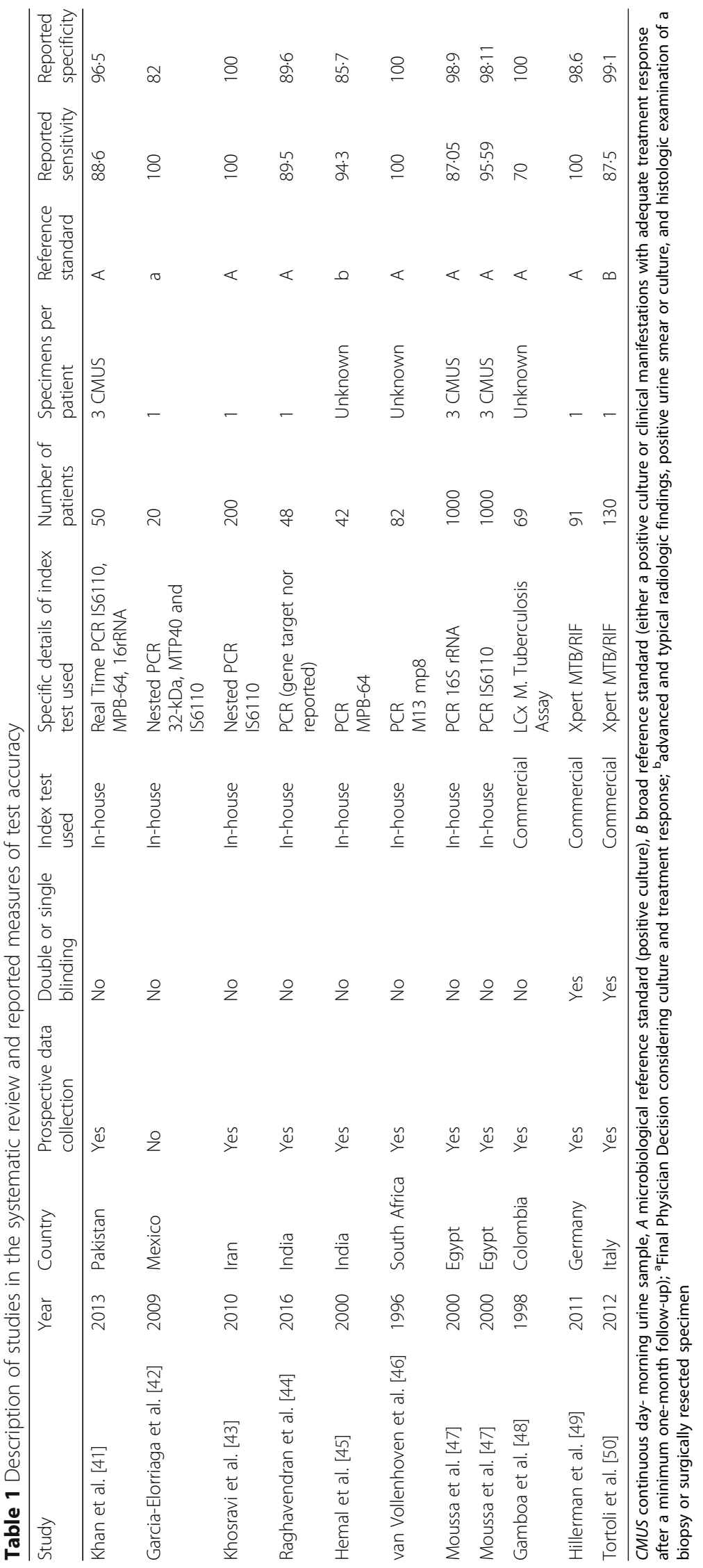


technique and two used a nested PCR technique; additionally, the gene target and primers were also different in each one.

\section{Quality appraisal}

Figure 2 shows the results of the quality evaluation using the QUADAS-2 tool; 64\% of the studies, all of which used PCR, were considered to be at high risk of bias in the index test and reference standard domains (see Additional file 1: Appendix S2 and Table S1 for detailed information). Within the index test and reference standard domain, studies were considered to be at high risk of bias because they did not blind operators to results of the reference standard or index test respectively, furthermore the protocols used in the "in-house" NAATs were not standardized and variations in technical aspects could have introduced bias.

For this latter reason, all the PCR studies were also considered as having a high concern of applicability in the index test domain. Additionally, 3 studies were considered as high concern of applicability in the reference standard domain because they used other than microbiological or broad reference standard.

\section{Summary measures}

Figure 3 shows the diagnostic accuracy of the studies distributed in three groups, PCR, LCR and Xpert MTB/ RIF with a calculated confidence interval (CI) of 95\% and a statistic continuity correction of $5 \%$.

Within the studies that used PCR there was a wide heterogeneity by assessing the forest plot (Fig. 3). Because the low study quality of these studies, the different protocols and variants of PCR used, and the visual heterogeneity assessed in the forest plots, we decided not to proceed with a summary measure. Regarding LCR, only one study addressed this test.

The two studies that used Xpert MTB/RIF showed good quality, and heterogeneity was further assessed by $X^{2}$ test. Table 2 shows the summary measures of these studies. We also plotted a SROC summary curve (Fig. 4). The summary sensitivity and specificity was $0.87(0.66-$ $0.96)$ and $0.91(0.84-0.95)$ respectively.

\section{Discussion}

Our systematic review indicates that Xpert MTB/RIF performance in urine samples for GUTB diagnosis showed sensitivity and specificity rates ranging from 0.83 to 0.95 and 0.79 to 0.99 , respectively. While these results show acceptable diagnostic accuracy, a proper interpretation of results should take into account the wide confidence intervals of the outcome variables, several sources of heterogeneity, such as quality issues, different target genes, different protocols, and different number of specimens analyzed. It should be noted that Xpert $\mathrm{MTB} / \mathrm{RIF}$ is a WHO recommended initial diagnostic test for all patients with sign and symptoms of TB [10].

Within the diverse protocols followed for PCR tests, possible sources of false positive and negative results are contamination and inhibition respectively. Contamination is usually caused by amplification products that get aerosolized and interfere with subsequent assays, this is especially true for nested PCR methods, in which repeated amplifications are done [27]. If not controlled, in a relatively short time, laboratory reagents, equipment, and even ventilation systems get contaminated with these products [27]. Metabolites, drugs, and other body fluid substances may produce inhibition of PCR; in urine, the most critical inhibitor is urea, which can lead to polymerase degradation in a concentration-dependent manner [28]. While doing PCR demands a controlled protocol to be followed carefully to avoid both inhibitors and contaminants, Xpert MTB/RIF does not face the same problem [29].

The pooled estimates of Xpert MTB/RIF studies in our analysis, showed satisfactory sensitivity and specificity: 0.87 and 0.91 respectively. However, only specificity had a narrow CI. Clinically it can be translated into the test correctly reporting $91 \%$ of patients

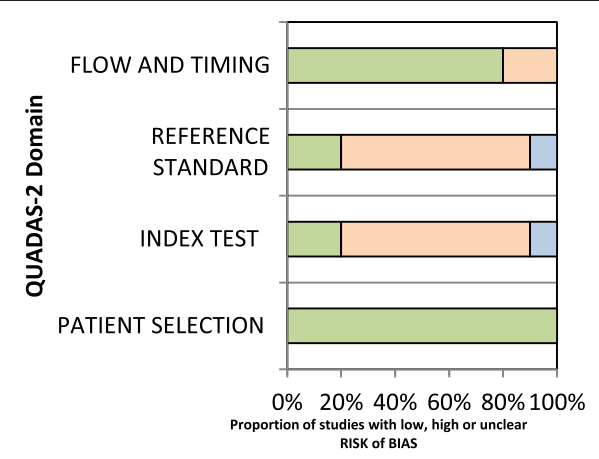

$\square$ Low $\square$ High $\square$ Unclear

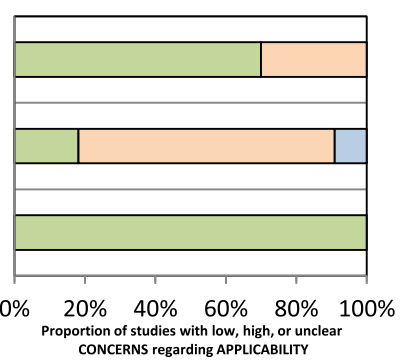

Fig. 2 Summary of Quality Assessment tool for Diagnostic Accuracy tests (QUADAS-2). The point estimates of sensitivity and specificity from each study are shown as solid circles. Error bars are 95\% confidence intervals. A statistical continuity correction of 5\% has been applied 


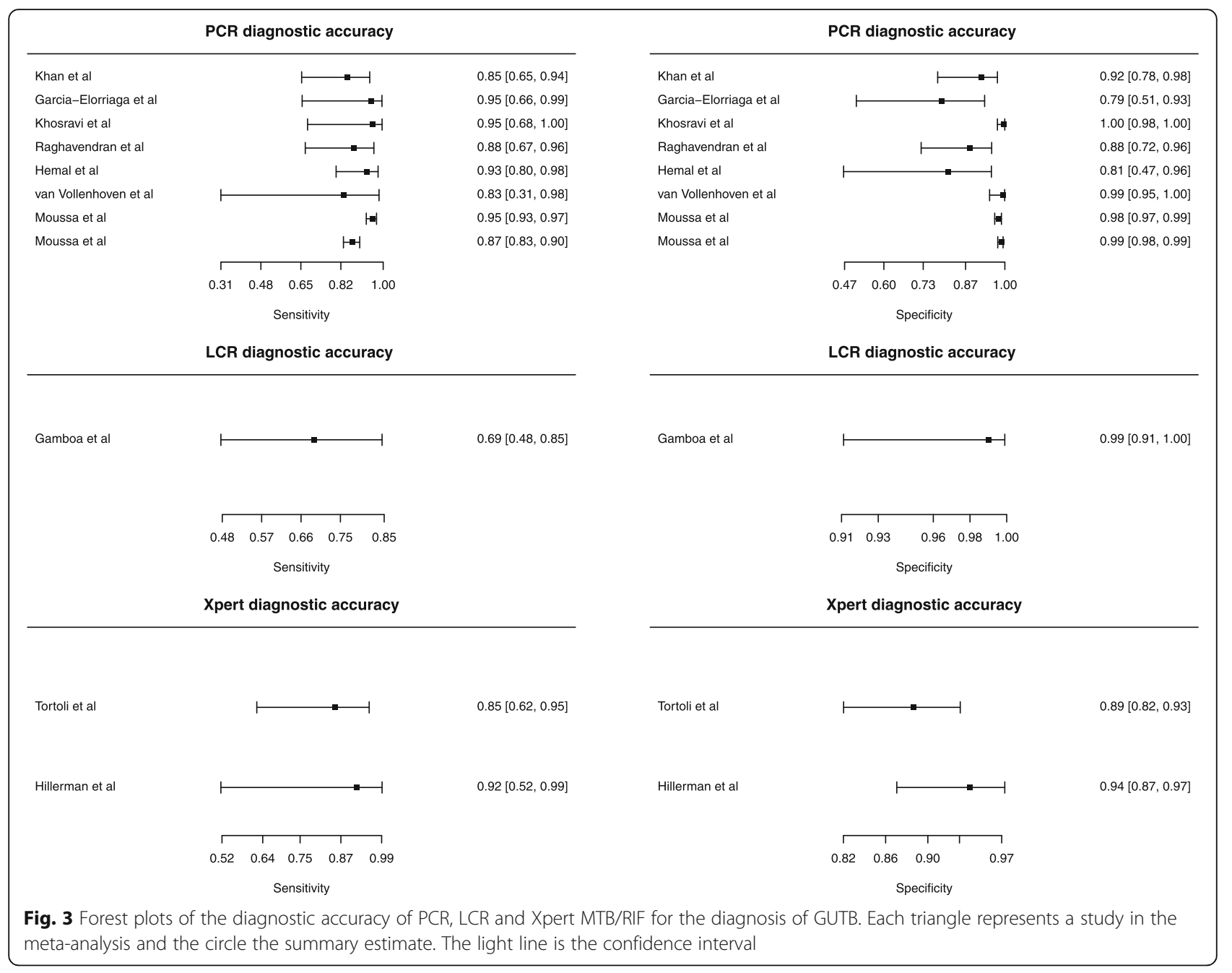

without the disease as true negative. Because this meta-analysis only comprised two studies, further research is required to upgrade and get more precise pooled estimates. Additionally, positive predictive values (PPV) and negative predictive values (NPV) which depend on the prevalence of the disease should be reported for different clinical settings as they have more practical utility.

Table 2 Summary measures of test accuracy of Xpert MTB/RIF studies

\begin{tabular}{lll}
\hline Test property & $\begin{array}{l}\text { Summary measure of test } \\
\text { accuracy }^{\text {a }}(95 \% \mathrm{Cl})\end{array}$ & $\begin{array}{l}\text { Test for heterogeneity } \\
\text { b-value }\end{array}$ \\
\hline Sensitivity & $0.87(0.66-0.96)$ & 1 \\
Specificity & $0.91(0.84-0.95)$ & 0.27 \\
Diagnostic odds ratio & $58.16(15.75-214.92)$ & 0.43 \\
LR+ & $9.66(4.12-19.2)$ & 0.31 \\
LR- & $0.15(0.04-0.40)$ & 0.36 \\
\hline
\end{tabular}

${ }^{\mathrm{a}}$ Random effects model ${ }^{\mathrm{b}} \mathrm{X}^{2}$ test for heterogeneity
Information of drug resistance among patients with GUTB is lacking [1, 2, 30, 31]. In this context, none of the Xpert MTB/RIF studies analyzed rifampicin resistance, possibly because they were done in high-income countries, where multi-drug resistant (MDR) tuberculosis is not usually a concern. One study in China used a different test, GenoType ${ }^{\circledast}$ MTBDRplus, which evaluates isoniazid and rifampicin resistance, in an outpatient setting [32]. They found that one-third of their patients had drug-resistant GUTB, and one-fourth had MDR-GUTB. Subsequent Xpert MTB/RIF or GenoType ${ }^{\curvearrowleft}$ MTBDRplus studies must help characterize drug resistance epidemiology while evaluating the clinical utility of these tests in different MDR prevalence settings.

We identified two main limitations on studies, the reference standard and the number of specimens analyzed were not the same for all, and they did not report if test accuracy changed in patients with comorbidities.

In the studies that used positive urine culture as reference standard, there is chance that true positives cases 


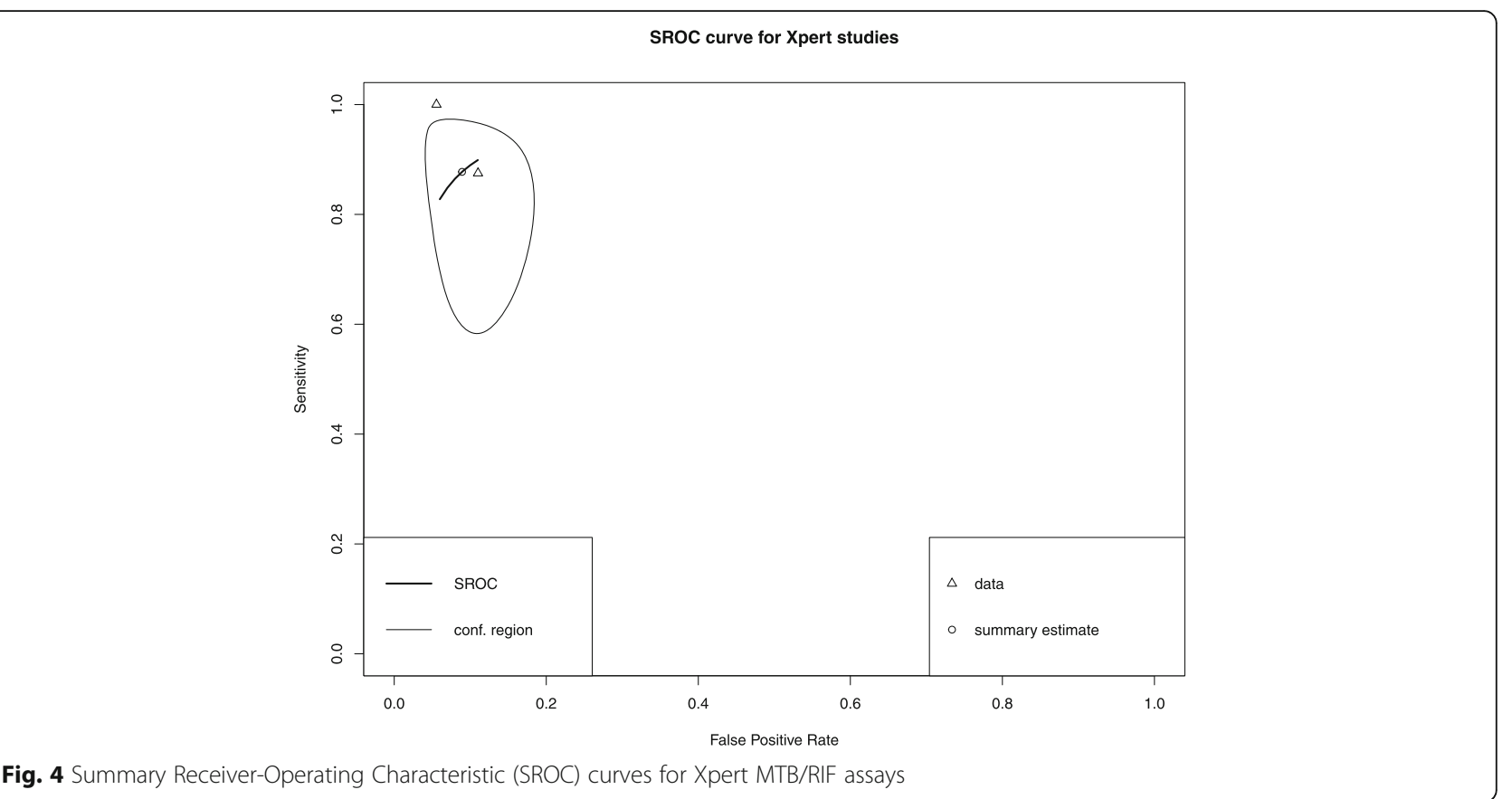

with only clinical response after treatment were not detected but tagged as false positives; rendering lower specificity. Additionally, some studies analyzed more than one specimen per patient. While there is a consensus based on an expert opinion for using three consecutive morning samples for culture or NAATs, there is no study that addresses this particular issue [33] Increasing the number of specimens can increase sensitivity at the cost of lowering specificity. In addition, it is still unknown if this strategy is cost-effective.

Comorbidities within the populations studied were not reported in any of the articles selected. We were especially concerned about HIV patients, as it has been shown that accuracy of diagnostic tests varies in this population. In HIV-infected patients, those presenting with pulmonary $\mathrm{TB}$ typically have a paucibacillary disease [34, 35]. However, in tuberculous meningitis, two studies reported higher Xpert MTB/RIF accuracy in those patients infected with HIV [36, 37]. We suggest that authors consider HIV-status in future research in GUTB patients.

\section{Strengths and weaknesses of the study}

We identified some weaknesses in our review. We could not assess male and female genital tuberculosis through this review because they lack a clear reference standard and thus, there would be a high concern of bias in the analysis [20]. We did not evaluate the effect of adding NAATs to other tests, and the net effect of NAATs on positive predictive values or negative predictive values since these depend on the prevalence of the disease.
Furthermore, we could not explore the effect of issues such as expertise with NAATs equipment, and laboratory infrastructure because of poor reporting.

Our study has several strengths: an exhaustive search strategy (including conference abstracts, and no language restriction), with more than 2000 patients evaluated and a deep critical quality appraisal, exploring heterogeneity in accordance with published guidelines, and following PRISMA recommendations for systematic reviews [22].

\section{Limitations}

The lack of quality reporting in diagnostic interferes with critical appraisal and replication of studies [38]. We encourage authors to follow the Standards for Reporting Diagnostic Accuracy (STARD) in order to achieve transparency, completeness and excellence of reporting [39]. The STARD was updated in 2015 [40], and comprises a list of 30 essential items for highquality diagnostic accuracy studies; it has also incorporated evidence about sources of bias and variability in diagnostic accuracy.

\section{Conclusion}

GUTB is an overlooked disease, an unspecific clinical picture and limited conventional tests account for this problem. Diagnosis is difficult and frequently delayed, leading to major impact on the urinary tract system.

All PCR studies were highly heterogeneous as they involved different gene targets, different protocols and had several quality concern issues. Consequently, no 
meta-analysis could be done. Xpert MTB/RIF studies, in contrast, were high quality reported and were not heterogeneous. Our analysis showed a favorable specificity. Studies with high-quality reporting are urgently needed to improve diagnosis of GUTB.

\section{Additional file}

\section{Additional file 1: Complete search strategy and details on quality} evaluation. (DOCX $18 \mathrm{~kb}$ )

\section{Abbreviations}

EPTB: Extrapulmonary tuberculosis; GUTB: Genitourinary tuberculosis; LCR: Ligase chain reaction; MDR: Multi-drug resistant; NAAT: Nucleic acid amplification test; NPV: Negative predictive values; PCR: Polymerase chain reaction; PPV: Positive predictive values; QUADAS-2: Quality Assessment Tool for Diagnostic Accuracy Studies; SROC: Summary receiver-operating curve; STARD: Standards for Reporting Diagnostic Accuracy; TB: Tuberculosis; UGTB: Urogenital tuberculosis; WHO: World Health Organization

\section{Acknowledgements}

The team wants to give special thanks to Seungseo Choi, a medical student from Facultad de Medicina Alberto Hurtado at UPCH, for his translation of Korean hits and manuscripts and to Maria Rueda, MD for her meaningful suggestions on the paper.

\section{Funding}

This study did not receive any funding. CU-G received support from the Program for Advanced Research Capacities for AIDS in Peru (PARACAS) at Universidad Peruana Cayetano Heredia (D43TW00976301) from Fogarty International Center at the U.S. National Institute of Health $(\mathrm{NIH})$.

\section{Availability of data and materials}

The datasets generated during and analysed during the current study are not publicly available but are available from the corresponding author on reasonable request.

\section{Authors' contributions}

CA and CU-G designed the study. CA and VO screened studies for inclusion. CA and CU-G wrote the report and analyzed data. CU-G, VO, CS, MM and LZ critically interpreted data and revised the report. All authors read and approved the final manuscript.

\section{Competing interests}

The authors declare that they have no competing interests.

\section{Consent for publication}

Not applicable.

\section{Ethics approval and consent to participate}

Not applicable.

\section{Publisher's Note}

Springer Nature remains neutral with regard to jurisdictional claims in published maps and institutional affiliations.

\footnotetext{
Author details

${ }^{1}$ Facultad de Medicina Alberto Hurtado, Universidad Peruana Cayetano Heredia, Av. Honorio Delgado 430, Urb. Ingeniería, S.M, P Lima, Perú. ${ }^{2}$ Department of Urology, Wake Forest University, Winston Salem, NC, USA ${ }^{3}$ Servicio de Urología, Hospital Nacional Cayetano Heredia, Lima, Perú. ${ }^{4}$ Instituto de Medicina Tropical Alexander von Humboldt, Universidad Peruana Cayetano Heredia, Lima, Peru. ${ }^{5}$ Departamento de Enfermedades Infecciosas, Tropicales y Dermatológicas, Hospital Nacional Cayetano Heredia, Lima, Peru. ${ }^{6}$ Department of Clinical Research, London School of Hygiene and Tropical Medicine, London, UK. ${ }^{7}$ Department of International Health, Johns Hopkins Bloomberg School of Public Health, Baltimore, USA.
}

Received: 12 September 2016 Accepted: 18 May 2017

Published online: 05 June 2017

\section{References}

1. World Health Organization (WHO). Global Tuberculosis Report. Ginebra: WHO. 2015:2015.

2. Figueiredo AA, Lucon AM, Junior RF, Srougi M. Epidemiology of urogenital tuberculosis worldwide. Int J Urol. 2008;15:827-32.

3. Kulchavenya E. Urogenital tuberculosis: definition and classification. Ther Adv Infect Dis. 2014;2:117-22.

4. Figueiredo AA, Lucon AM. Urogenital tuberculosis: update and review of 8961 cases from the world literature. Rev Urol. 2008;10:207-17.

5. Molina RL, Diouf K, Nour NM. Tuberculosis and the obstetriciangynecologist: a global perspective. Rev Obstet Gynecol. 2013;6:174-81.

6. Abbara A, Davidson RN. Etiology and management of genitourinary tuberculosis. Nat Rev Urol. 2011;8:678-88.

7. Bultitude MF. Campbell-Walsh Urology tenth edition. BJU Int. 2012;109:E10.

8. Kapoor R, Ansari MS, Mandhani A, Gulia A. Clinical presentation and diagnostic approach in cases of genitourinary tuberculosis. Indian J. Urol. 2008:24:401-5.

9. Tostmann A, Kik SV, Kalisvaart NA, Sebek MM, Verver S, Boeree MJ, et al. Tuberculosis transmission by patients with smear-negative pulmonary tuberculosis in a large cohort in the Netherlands. Clin. Infect. Dis. 2008:47:1135-42.

10. World Health Organization. Policy framework for implementing new tuberculosis diagnostics. Geneva: World Health Organization; 2011.

11. Ling DI, Flores LL, Riley LW, Pai M. Commercial nucleic-acid amplification tests for diagnosis of pulmonary tuberculosis in respiratory specimens: meta-analysis and meta-regression. PLoS One. 2008;3:e1536.

12. Adelman MW, Kurbatova E, Wang YF, Leonard MK, White N, McFarland DA, et al. Cost analysis of a nucleic acid amplification test in the diagnosis of pulmonary tuberculosis at an urban hospital with a high prevalence of TB/HIV. PLoS One. 2014;9:e100649.

13. Kobayashi M, Ray SM, Hanfelt J, Wang YF. Diagnosis of tuberculosis by using a nucleic acid amplification test in an urban population with high HIV prevalence in the United States. PLoS One. 2014;9:e107552.

14. Taegtmeyer M, Beeching NJ, Scott J, Seddon K, Jamieson S, Squire SB, et al. The clinical impact of nucleic acid amplification tests on the diagnosis and management of tuberculosis in a British hospital. Thorax. 2008:63:317-21.

15. Marks SM, Cronin W, Venkatappa T, Maltas G, Chon S, Sharnprapai S, et al. The health-system benefits and cost-effectiveness of using Mycobacterium tuberculosis direct nucleic acid amplification testing to diagnose tuberculosis disease in the United States. Clin Infect Dis Off Publ Infect Dis Soc Am 2013:57:532-42.

16. Centers for Disease Control and Prevention (CDC). Updated guidelines for the use of nucleic acid amplification tests in the diagnosis of tuberculosis. MMWR Morb. Mortal. Wkly. Rep. 2009;58:7-10.

17. Celik C, Gozel MG, Bakici MZ, Berk S, Ozsahin SL, Gulturk E. Applicability of Xpert MTB/RIF assay for routine diagnosis of tuberculosis: a four-year singlecenter experience. Turk J Med Sci. 2015:45:1329-34.

18. Theron G, Peter J, Richardson M, et al. The diagnostic accuracy of the GenoType ${ }^{\oplus}$ Mtbdrsl assay for the detection of resistance to second-line antituberculosis drugs. Cochrane Database Syst Rev. 2014;10:4-123.

19. Shah HN Badlani GH. Genitourinary tuberculosis. An Update Curr Bladder Dysfunct Rep. 2013;8:186-96.

20. Bose M. Female genital tract tuberculosis: how long will it elude diagnosis? Indian J Med Res. 2011;134:13-4.

21. Macaskill P, Gatsonis C, Deeks JJ, Harbord RM, Takwoingi Y. Analysing and presenting results. In: Deeks J, Bossuyt P, Gatsonis C, eds. Cochrane handbook for systematic reviews of diagnostic test accuracy. Version 0.9.0. London: The Cochrane Collaboration; 2010.

22. Moher D, Liberati A, Tetzlaff J, Altman DG, The PRISMA Group. Preferred Reporting Items for Systematic Reviews and Meta-Analyses: the PRISMA statement. PLoS Med. 2009;6:e1000097.

23. Whiting PF, Rutjes AWS, Westwood ME, Mallett S, Deeks JJ, Reitsma JB, et al. QUADAS-2: a revised tool for the quality assessment of diagnostic accuracy studies. Ann Intern Med. 2011;155:529-36.

24. Core Team R. R Foundation for Statistical Computing: Vienna, Austria, R: A Language and Environment for Statistical Computing. 2016; Available at http://www.R-project.org 
25. Doebler P. Meta-Analysis of Diagnostic Accuracy with mada. 2012; Available at https://cran.r-project.org/package=mada

26. Reitsma JB, Glas AS, Rutjes AWS, Scholten RJPM, Bossuyt PM, Zwinderman AH. Bivariate analysis of sensitivity and specificity produces informative summary measures in diagnostic reviews. J Clin Epidemiol. 2005;58:982-90.

27. Aslanzadeh J, Preventing PCR. Amplification carryover contamination in a clinical laboratory. Ann Clin Lab Sci. 2004;34:389-96.

28. Schrader C, Schielke A, Ellerbroek L, Johne R. PCR inhibitors - occurrence, properties and removal. J Appl Microbiol. 2012;113:1014-26.

29. Banada PP, Sivasubramani SK, Blakemore R, Boehme C, Perkins MD, Fennelly K, et al. Containment of bioaerosol infection risk by the Xpert MTB/RIF assay and its applicability to point-of-care settings. J Clin Microbiol. 2010;48:3551-7.

30. Zajaczkowski T. Genitourinary tuberculosis: historical and basic science review: past and present. Cent Eur J Urol. 2012;65:182-7.

31. Merchant S, Bharati A, Merchant N. Tuberculosis of the genitourinary system-urinary tract tuberculosis: renal tuberculosis-part I. Indian J Radiol Imaging. 2013;23:46-63.

32. Ye Y, Hu X, Shi Y, Zhou J, Zhou Y, Song X, et al. Clinical Features and DrugResistance Profile of Urinary Tuberculosis in South-Western China: a Crosssectional Study. Medicine (Baltimore). 2016;95:e3537.

33. Diagnostic Standards and Classification of Tuberculosis in Adults and Children. This official statement of the American Thoracic Society and the Centers for Disease Control and Prevention was adopted by the ATS Board of Directors, July 1999. This statement was endorsed by the Council of the Infectious Disease Society of America, September 1999. Am J Respir Crit Care Med 2000; 161: 1376-1395.

34. Swaminathan S, Padmapriyadarsini C, Narendran G. HIV-associated tuberculosis: clinical update. Clin Infect Dis. 2010;50:1377-86.

35. Steingart KR, Schiller I, Horne DJ, Pai M, Boehme CC, Dendukuri N. Xpert ${ }^{\circledast}$ MTB/RIF assay for pulmonary tuberculosis and rifampicin resistance in adults. Cochrane Database Syst Rev. 2014;1:CD009593.

36. Patel $V B$, Theron $G$, Lenders $L$, et al. Diagnostic accuracy of quantitative $P C R$ (Xpert MTB/RIF) for Tuberculous meningitis in a high burden setting: a prospective study. PLoS Med. 2013;10 doi:10.1371/journal.pmed.1001536.

37. Nhu NTQ, Heemskerk D, Thu DDA, Chau TTH, Mai NTH, Nghia HDT, et al. Evaluation of GeneXpert MTB/RIF for diagnosis of Tuberculous meningitis. J Clin Microbiol. 2014;52:226-33.

38. Korevaar DA, Enst WA van, Spijker R, Bossuyt PMM, Hooft L. Reporting quality of diagnostic accuracy studies: a systematic review and meta-analysis of investigations on adherence to STARD. Evid Based Med 2014;19:47-54.

39. Bossuyt PM, Reitsma JB, Bruns DE, Gatsonis CA, Glasziou PP, Irwig LM, et al. The STARD statement for reporting studies of diagnostic accuracy: explanation and elaboration. Ann Intern Med. 2003;138:W1-12.

40. Bossuyt PM, Reitsma JB, Bruns DE, Gatsonis CA, Glasziou PP, Irwig L, et al. STARD 2015: an updated list of essential items for reporting diagnostic accuracy studies. BMJ. 2015;351:h5527.

41. Khan F, Cheema F, Khan M. Accuracy of urinary PCR as compared with urine culture for early diagnosis of genitourinary tuberculosis. Pak J Med Health Sci. 2013;7:675-8

42. Garcia-Elorriaga G, Gracida-Osorno C, Carrillo-Montes G, Gonzalez-Bonilla C. Clinical usefulness of the nested polymerase chain reaction in the diagnosis of extrapulmonary tuberculosis. Salud Publica Mex. 2009;51:240-5.

43. Khosravi AD, Hashemzadeh M, Ghorbani A, Seghatoleslami S. Comparison of nested-PCR technique and culture method in detection of Mycobacterium tuberculosis from patients suspected to genitourinary tuberculosis. Afr J Biotechnol. 2010;9:2151-5.

44. Raghavendran M, Venugopal A. MP24-02 PCR in GENITO urinary tuberculosis. J Urol. 2016;195:e270-1.

45. Hemal AK, Gupta NP, Rajeev TP, Kumar R, Dar L, Seth P. Polymerase chain reaction in clinically suspected genitourinary tuberculosis: comparison with intravenous urography, bladder biopsy, and urine acid fast bacilli culture. Urology. 2000;56:570-4.

46. van Vollenhoven P, Heyns CF, de Beer PM, Whitaker P, van Helden PD, Victor T. Polymerase chain reaction in the diagnosis of urinary tract tuberculosis. Urol Res. 1996;24:107-11.

47. Moussa OM, Eraky I, El-Far MA, Osman HG, Ghoneim MA. Rapid diagnosis of genitourinary tuberculosis by polymerase chain reaction and nonradioactive DNA hybridization. J Urol. 2000;164:584-8.

48. Gamboa F, Dominguez J, Padilla E, Manterola JM, Gazapo E, Lonca J, et al. Rapid diagnosis of extrapulmonary tuberculosis by ligase chain reaction amplification. J Clin Microbiol. 1998;36:1324-9.
49. Hillemann D, Rusch-Gerdes S, Boehme C, Richter E. Rapid molecular detection of extrapulmonary tuberculosis by the automated GeneXpert MTB/RIF system. J Clin Microbiol. 2011;49:1202-5.

50. Tortoli E, Russo C, Piersimoni C, Mazzola E, Dal Monte P, Pascarella M, et al. Clinical validation of Xpert MTB/RIF for the diagnosis of extrapulmonary tuberculosis. Eur Respir J. 2012;40:442-7.

\section{Submit your next manuscript to BioMed Central and we will help you at every step:}

- We accept pre-submission inquiries

- Our selector tool helps you to find the most relevant journal

- We provide round the clock customer support

- Convenient online submission

- Thorough peer review

- Inclusion in PubMed and all major indexing services

- Maximum visibility for your research

Submit your manuscript at www.biomedcentral.com/submit
) Biomed Central 